\title{
Reliability-Based Analysis of Sight Distance Modelling for Traffic Safety
}

\author{
César de Santos-Berbel, ${ }^{1}$ Mohamed Essa, ${ }^{2}$ Tarek Sayed, ${ }^{2}$ and María Castro1 \\ ${ }^{1}$ Departamento de Ingeniería Civil: Transporte y Territorio, Technical University of Madrid (UPM), Madrid, Spain \\ ${ }^{2}$ Department of Civil Engineering, University of British Columbia, 6250 Applied Science Lane, Vancouver, BC, Canada V6T $1 Z 4$ \\ Correspondence should be addressed to César de Santos-Berbel; cesar.desantos@upm.es
}

Received 6 March 2017; Revised 20 April 2017; Accepted 3 May 2017; Published 31 May 2017

Academic Editor: Alexandre G. De Barros

Copyright (c) 2017 César de Santos-Berbel et al. This is an open access article distributed under the Creative Commons Attribution License, which permits unrestricted use, distribution, and reproduction in any medium, provided the original work is properly cited.

\begin{abstract}
Sight distance is of the utmost importance for traffic safety. The consideration of three-dimensional (3D) available sight distance (ASD) in geometric design has been supported by several researchers. However, existing ASD estimation methods are twodimensional (2D) in nature, which do not evaluate varying visibility conditions. This paper compares different methodologies of modelling the ASD. The ASD of 402 horizontal curves, located in twelve in-service two-lane rural highways, was analyzed. Three ASD estimation methods were used which include a 2D method and two different 3D methods. The ASD results obtained through 2D and 3D methodologies are compared. Also, the different conditions of the existing roadside features or geometric elements, under which the 3D ASD estimation is important, were identified. Next, reliability theory is utilized to evaluate the risk level (probability of noncompliance, $P_{\mathrm{nc}}$ ) associated with limited sight distance for each ASD modelling method. The results of the comparison emphasized the importance of considering the 3D modelled sight distance when evaluating the associated risk either in highway design or during the service life. In addition, the results indicated that the ASD modelling approach can have a significant impact on the estimation of the safety of highway design.
\end{abstract}

\section{Introduction}

The importance of providing adequate sight distance for a safe and efficient traffic is recognized by researchers and most standard design manuals $[1,2]$. At any point on a highway, the available sight distance (ASD) should be sufficient to allow drivers to safely control their vehicles. In design practice, the ASD is compared to the required sight distance for various driving and controlling tasks (e.g., stopping, passing, decision, and at intersections).

ASD is defined as the section of highway ahead that is visible to the driver, which is measured along the path travelled by the vehicle. Geometric design guides usually propose two-dimensional (2D) sight distance models, which fall short when the alignment is composed of complex sequences of three-dimensional (3D) elements. As those models may underestimate or overestimate the actual ASD, a $3 \mathrm{D}$ procedure to evaluate sight distance is preferable. The ASD depends on many parameters such as cross-section and roadside elements, besides the vertical and horizontal alignments. Furthermore, the ASD may vary due to maintenance actions often affecting the original highway geometrics. Also, the presence of new elements such as vegetation or buildings may limit the ASD after construction. A regular evaluation of ASD is thus necessary to ensure that standards are met during the service life of such transportation facilities.

This paper compares different methodologies of modelling the ASD. The ASD of 402 horizontal curves, located in twelve in-service two-lane rural highways, was analyzed. Three ASD estimation methods were used which include a 2D method and two different 3D methods. The 3D modelling approaches utilize Light Detection And Ranging (LiDAR) data, which provides comprehensive and more accurate input data to study sight distance. The ASD results obtained through $2 \mathrm{D}$ and $3 \mathrm{D}$ methodologies are compared. Also, the different conditions of the existing roadside features or geometric elements, under which the 3D ASD estimation is important, were identified. 


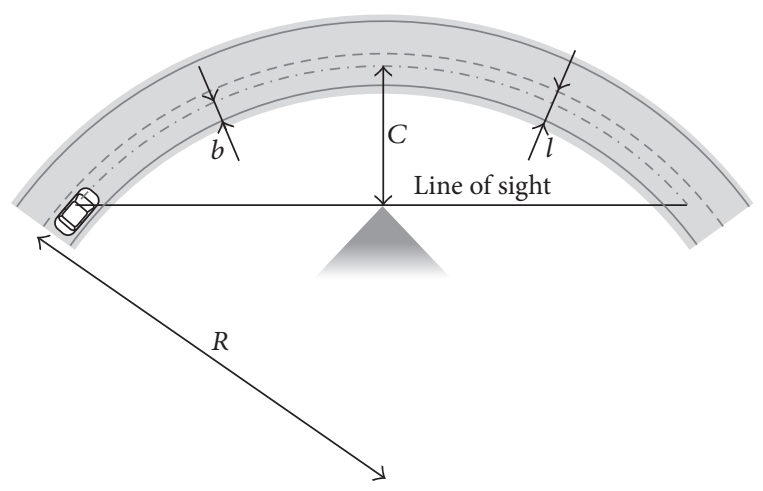

FIGURE 1: 2D modelling of ASD on horizontal curves longer than ASD.

Next, reliability theory is utilized to evaluate the risk level associated with limited sight distance for each ASD modelling method. Reliability analysis has been recently advocated as an effective approach to account for uncertainty in the geometric design process and to evaluate the risk associated with a specific design. In this approach, the design parameters are considered as random variables expressed in terms of their probability distributions as opposed to single value estimations in the deterministic approach. Design equations are represented as limit-state functions (LSF). The LSF represents the difference between the supply (ASD) and the demand (SSD) (i.e., LSF $=$ ASD - SSD). If the SSD exceeds the ASD (i.e., LSF < 0), the design is considered to be failed or not complied with the standard design requirements. Reliability theory is used to evaluate the probability of noncompliance $\left(P_{\mathrm{nc}}\right)$ which is associated with a measure of probability that the SSD exceeds the ASD or that a specific design does not meet standard requirements [3-5]. Finally, $P_{\mathrm{nc}}$ results obtained through the 2D and the 3D ASD were compared in order to investigate the significance of the safety implications of the ASD modelling approach.

\section{Background}

2.1. $2 D$ and $3 D$ Available Sight Distance Estimations. ASD estimation methods found in literature can be classified into two groups: detached 2D methods for horizontal and vertical alignment and 3D methods. 2D approaches have traditionally been used in geometric design and are presented in most current standards. For example, the Spanish standard on geometric design [6] provides separate 2D methods for the estimation of ASD on the horizontal and vertical alignment. On the horizontal projection, such a standard demands a minimum lateral clearance on curves $C$, which is given by the following expression:

$$
C=(R-l)-(R-l+b) \cdot \cos \left(\frac{\mathrm{ASD}}{2 \cdot(R-l+b)}\right)
$$

where $R$ is the radius of the circular arc on the centerline, $l$ is the lane width, and $b$ is the offset between the observer's path and the roadway edge on the inner roadside (Figure 1).
When the clearance is known, the formula can be rewritten to estimate the ASD as follows:

$$
\mathrm{ASD}=2 \cdot(R-l+b) \cdot \cos ^{-1}\left(\frac{R-l-C}{R-l+b}\right) .
$$

In a similar way, the North American guides on geometric design $[1,7]$ provide a method to calculate the minimum ASD on simple horizontal curves. However, the analytical formula of the lateral clearance proposed in these guides applies only to circular curves longer than the sight distance and when both vehicle and sight obstructions are located within the limits of the horizontal curve circular arc. Otherwise, the results will be approximate and it would be advisable, according to these guidelines, to check the design by a graphical procedure or a computational method. Mauga et al. [8] reported the difficulties inherent to the use of these graphical methods and proposed a fully analytical model for determining the clearance boundary on a horizontal curve. Lovell [9] devised a method for automatic computation of ASD subject to constraints of horizontal geometry where precise information of sight obstructions is not available.

Several researchers agreed on the limited validity of 2D sight distance estimation methods. The ASD may vary depending on the highway horizontal and vertical geometry. Even on flat alignments, 2D horizontal ASD may still fluctuate depending on cross-section and roadside configuration and features. The literature shows that $2 \mathrm{D}$ models tend to either underestimate or overestimate ASD [10]. In addition, several studies have highlighted the need for considering 3D ASD in geometric design [11, 12]. Therefore, 3D ASD estimation procedures are gaining interest among researchers. Some procedures are focused on theoretical alignments at the design stage to provide drivers with adequate sight distance conditions. Hassan et al. [13] developed an analytical model to evaluate the effects of considering $3 \mathrm{D}$ alignments on design requirements for ASD. Ismail and Sayed [10] devised an algorithm for an accurate and efficient 3D estimation of ASD. The latter study enabled a precise study of the differences between estimating the vertical 2D ASD, the horizontal 2D ASD, and the 3D ASD. For design standards, the German recommendations for the visualization of roads [14] put forward a comprehensive 3D methodology to estimate ASD.

In addition, research on ASD estimation on in-service highways also met success. Castro et al. [15] developed a procedure based on geographic information systems (GIS) to estimate the ASD on highways where design data are either not available or not reliable. With this procedure, it is possible to use the trajectory data of a vehicle on a highway obtained with a Global Positioning System (GPS) device to determine the ASD. Castro et al. [16] devised an enhanced procedure to fully characterize sight distance conditions on in-service highways based on line-of-sight loops. Several ASD studies utilized LiDAR data to model the roadway and its roadsides [17-19]. This surveying technique successfully overcomes the lack of information of sight obstructions.

2.2. Available Sight Distance and Road Safety. Considerable research has been undertaken out to link visibility conditions and safety. On the one hand, the bare ASD or SSD values 
have been compared to accident data. Olson et al. [20] compared accident occurrence between crest vertical curves where ASD is limited and control sections meeting the SSD standards in Michigan. They concluded that there are significantly fewer accidents at sites where ASD is adequate. Fink and Krammes [21] stated that ASD explains some of the variability in crash frequency accounting for the speed reduction when approaching curves. Steinauer et al. [22] found correlation between ASD increase and accident rate reduction at horizontal curves on the Bavarian motorways. However, Fitzpatrick et al. [23] reported that limited ASD does not necessarily pose a safety problem. Such disagreement highlights the difficulties when addressing the safety implications of deficient ASD. Therefore, reliability has been advocated as an auxiliary technique to bridge the gap between ASD and safety. In this regard, safety performance functions may incorporate the reliability outcome to confirm the influence of insufficient ASD on accident frequency. Ibrahim and Sayed [24] met success in incorporating reliability risk measures for limited ASD in safety performance functions.

2.3. Reliability Theory. The term reliability refers to the complement of the failure probability (3). In the reliability analysis, the term probability of failure represents the probability of an undesired event exceeding a certain threshold. In road design, researchers have proposed the use of $P_{\mathrm{nc}}$ to label the probability of a design that does not meet standard $[3-5,25]$.

$$
\text { Reliability }=1-P_{\mathrm{nc}} \text {. }
$$

Reliability analysis has two components: random variables that describe the uncertainty and LSF that define the failure mode. The first step is defining a LSF, denoted by $g(x)$, which defines what is considered to be noncompliance where $x$ is the input vector of random variables [26]. Generally, the LSF is represented as a balance between supply and demand. For example, the supply is the ASD, and the demand is the required SSD. $P_{\mathrm{nc}}$ is given by (5), in which $f(x)$ is the joint probability distribution function for random variables [26].

$$
\begin{gathered}
g(x) \leq 0 \Longleftrightarrow \text { Failure or noncompliance, } \\
P_{\mathrm{nc}}=P(g \leq 0)=\iint_{g(x) \leq 0} \cdots \int f(x) d x .
\end{gathered}
$$

\subsection{Reliability Applications in Transportation Engineering.} Several studies utilized reliability analysis in transportation engineering applications. Chen et al. [27] modelled alternate capacity reliability measures in transportation networks. Ben-Akiva et al. [28] proposed a probabilistic costbased highway design. Faghri and Demetsky [29] used a probabilistic approach to evaluate the limitation in sight distance at road and railway grade crossings. Easa [30] proposed a reliability-based design of sight distance at railroad grade crossings. Easa [31] applied a reliability method in order to evaluate sight distance at intersections. Navin [25] utilized a partial safety factor to propose a model for geometric design. Also, Navin [32] devised a method to estimate the margin of safety and reliability index for isolated highway components using the SSD to demonstrate the method.

Echaveguren et al. [33] proposed a methodology based on the reliability theory to determine the margin of safety of an existing horizontal curve. Richl and Sayed [4] applied First-Order Reliability Method (FORM) to evaluate the safety risk of narrow medians combined with tight horizontal curves. Regarding sampling methods, Khoury and Hobeika [34] used Monte Carlo simulation to estimate the risk level of passing maneuvers based on the probability of inadequate passing sight distance (PSD) and the consequence of a collision. Sarhan and Hassan [35] used Monte Carlo simulation to estimate the probability of noncompliance for three-dimension sight distance on overlapped horizontal and vertical curves. De Santos-Berbel and Castro [36] performed a 3D full-length evaluation of SSD through a Monte Carlo simulation.

Reliability analysis has been utilized in guide calibration in order to yield consistent safety levels for design. Ismail and Sayed [37] introduced a general framework for calibrating standard design models and determining target values for design safety. Also, Ismail and Sayed [37] investigated the safety implications of sight distance limitation on road segments and the risk associated with deviation from standards. Furthermore, Ismail and Sayed [38] presented a decision mechanism that enables the efficient use of available right-of-way for new highway construction with restricted sight distance to minimize the overall risk of the design. Ibrahim et al. [39] presented a methodology to select a suitable combination of cross-section elements with restricted sight distance to yield reduced collisions and consistent risk levels. Hussein et al. [5] investigated the calibration of geometric design models to yield consistent risk levels. Llorca et al. [40] developed a reliability analysis for PSD based on observation of maneuvers in two-lane roads.

Himes and Donnell [41] developed a probabilistic approach to the design of horizontal curves considering the effects of wet pavements and tire characteristics for passenger cars and heavy trucks. Musunuru and Porter [42] applied reliability analysis to evaluate the probability distribution of operational performance associated with decisions made to achieve a design level of service for number of lanes on a freeway. You et al. [43] used different performance functions for calculating the probability of vehicle failure modes (skidding and rollover at horizontal curves). You and Sun [44] established a dynamic simulation model, considering threedimensional alignment, for reliability analysis of vehicle stability on the combined horizontal and vertical curve. Shin and Lee [45] presented a reliability-based analysis to assess vehicle safety on horizontal curves based on vehicle dynamics on windy environments. Essa et al. [3] proposed the use of system reliability-based design of horizontal curves using several modes of failure, namely, SSD and vehicle skidding. Osama et al. [46] used reliability analysis framework to evaluate the risk of limited sight distance for permitted leftturn movements due to the presence of opposing left-turn vehicles. 
TABLE 1: Main features of the selected highways.

\begin{tabular}{|c|c|c|c|c|c|}
\hline Highway & Length $(\mathrm{km})$ & Terrain & Roadway width (m) & Paved width (m) & Number of curves \\
\hline M-104 & 10.9 & Rolling & 6.5 & 7.5 & 40 \\
\hline M-221 & 7.9 & Rolling & 6 & 7 & 20 \\
\hline M-222 & 5.4 & Rolling & 6 & 7 & 15 \\
\hline M-325 & 15.1 & Rolling & 5.5 & 5.5 & 54 \\
\hline M-513 & 9.1 & Rolling & 7 & 9 & 26 \\
\hline M-600 & 16.8 & Rolling & 7 & 9 & 30 \\
\hline \multirow{2}{*}{ M-601 } & \multirow{2}{*}{10.4} & Rolling & 7 & 11 & 19 \\
\hline & & Hilly & 6.5 & 7.5 & 6 \\
\hline M-607 & 12.3 & Rolling & 7 & 12 & 25 \\
\hline M-610 & 8.3 & Rolling & 6.5 & 7.5 & 28 \\
\hline M-611 & 5.1 & Rolling & 6.5 & 7.5 & 12 \\
\hline M-629 & 15.3 & Mountainous & 5 & 5 & 106 \\
\hline M-633 & 6.2 & Rolling & 5.5 & 6 & 21 \\
\hline Total & & & & & 402 \\
\hline
\end{tabular}

\section{Data Description}

This study was performed on twelve two-lane rural highways located in the Region of Madrid, Spain (Figure 2). The overall length of all sections is $122.7 \mathrm{~km}$ and their design speed ranges from 40 to $100 \mathrm{~km} / \mathrm{hr}$. 402 horizontal curves were selected for analysis. Considering both directions of these curves results in 804 cases for analysis. In the selection process, compound curves and low-deflection angle curves were removed from the sample. Detailed features of all selected highways can be found in Table 1.

\section{Methodology}

The analysis methodology consists of three main parts: (1) estimating the ASD of the selected highway segments using three different methods which include a $2 \mathrm{D}$ method and two different 3D methods; (2) determining the operating speed profile using speed prediction models; and (3) applying reliability theory to evaluate the risk level $\left(P_{\mathrm{nc}}\right)$ associated with limited sight distance for each ASD modelling method. More details of the analysis procedure are presented in the following subsections.

4.1. Available Sight Distance Estimation. Three ASD modelling methods were used in this study, a 2D method and two $3 \mathrm{D}$ methods. On each of the three methods, the parameters set by the Spanish standard [7] were used.

4.1.1. Two-Dimensional (2D) Estimation of the ASD. The horizontal 2D ASD was measured graphically on the horizontal projection of each horizontal curve using CAD software. The lateral clearance was assumed to be a constant value that equals the total width of both shoulder and ditch. Equation (2) was not used in this study to calculate the horizontal ASD, since the circular arc length of some horizontal curves is less than the calculated value of the ASD. On the other hand, the vertical 2D ASD was estimated analytically on crest vertical curves. In case that the crest curve length is longer than the
ASD, the ASD value was estimated by (6); otherwise, (7) was used [47]. Finally, the 2D ASD of each curve was determined to be the minimum of the horizontal 2D ASD and the vertical 2D ASD.

$$
\begin{aligned}
& \text { If } \mathrm{ASD}> \\
& \qquad \mathrm{ASD}_{\text {long }}=\sqrt{2 \cdot K_{V}} \cdot\left(\sqrt{h_{1}}+\sqrt{h_{2}}\right) ; \\
& \text { if } \mathrm{ASD}< \\
& \quad \mathrm{ASD}_{\text {short }}=-\frac{K_{V}}{L}\left(\sqrt{h_{1}}+\sqrt{h_{2}}\right)^{2}+\frac{L}{2},
\end{aligned}
$$

where $K_{V}$ is radius of the osculating circle of the crest parabola at its vertex $(\mathrm{m}) . h_{1}$ is driver's eye height above roadway surface $(1.1 \mathrm{~m})$ [6]. $h_{2}$ is target height above roadway surface $(0.2 \mathrm{~m})$ [6]. $L$ is length of vertical curve $(\mathrm{m})$.

4.1.2. Three-Dimensional (3D) Estimation of the ASD. The 3D modelling of the ASD can be considered more realistic than the $2 \mathrm{D}$ method. GIS-based software to estimate the 3D ASD was presented in previous studies $[16,19]$. The essential inputs to compute sight distance comprise a digital elevation model (DEM), either the highway centerline or vehicle paths, and driver's eye and target heights.

A DEM is a 3D depiction of the terrain's surface and, occasionally, other landscape features, through a collection of points or linear elements that shape terrain elevations. Current techniques are capable of producing high-resolution DEMs through surveying the study location using LiDAR equipment. There are two types of DEMs: (1) digital terrain models (DTMs) and (2) digital surface models (DSMs). The main difference between the two DEMs types is that DTMs represent the bare ground surface only, while DSMs consider both the bare ground surface and the relevant landscape elements above the terrain (e.g., vegetation, walls, and buildings). Hence, DSMs must yield lower values of the estimated 3D ASD. The differences in ASD results between the two DEM types were investigated by Castro et al. [19]. 


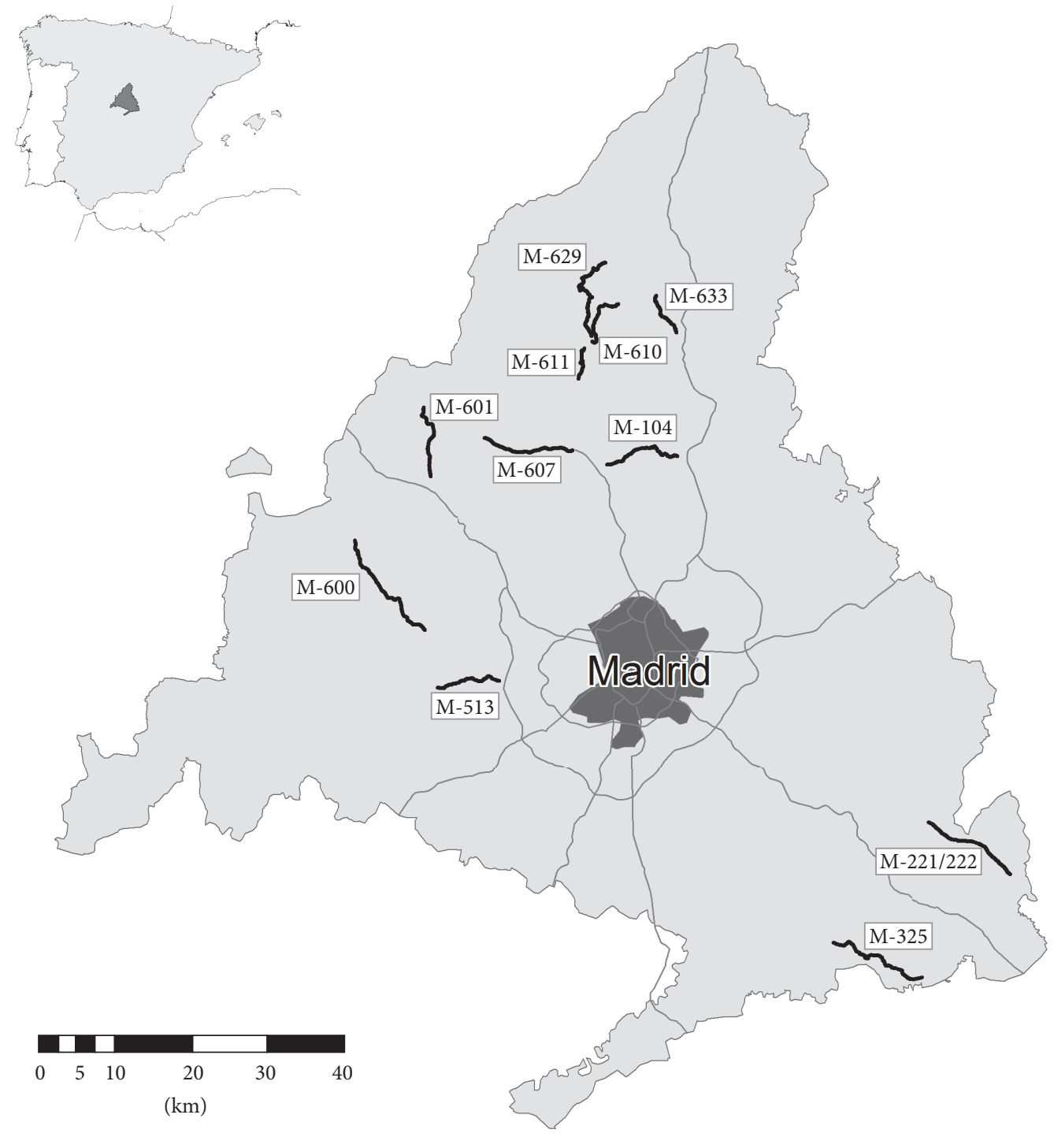

Selected highways

Figure 2: Location of selected highway segments.

In this study, both DTMs and DSMs are used in order to estimate the 3D ASD. The source of both elevation models was airborne LiDAR surveying. Using this surveying technique, a cloud of points for each highway segment was obtained with a resolution of one point per square meter.

Although the surveying procedure was the same for both DTMs and DSMs, the two types differ in terms of the data processing method used to depict the terrain surface. Thus, for each highway segment, two triangular irregular networks (TIN) were built up using the cloud of points obtained by surveying. The first TIN, which represents the DTM, only considered the topography of the bare ground surface of the highway segment and its roadsides. On the other hand, the second TIN, which constitutes the DSM, comprised the bare ground surface of the highway segment and its roadsides in addition to the relevant landscape elements above the terrain (such as vegetation).
For each highway segment, GIS-based software developed by Castro et al. [16] was used to compute the 3D ASD DTM and the 3D ASD DSM on each curve located in this highway segment, using the previously built networks: TIN DTM and TIN DSM, respectively. Within this software, the path of vehicle was set to be parallel to the outer lane border at an offset of 1.5 meter, which is conforming to the Spanish standard [6]. The path of vehicle was discretized into stations spaced one meter apart. The driver's eye height was set at $1.1 \mathrm{~m}$ and the target at a height of $0.2 \mathrm{~m}$, also in accordance with the same standard [6]. A virtual driver eye was successively placed on the path stations. For each driver's eye station, iterative trials were conducted to estimate the ASD at this station. In each trial, the target was placed on one of the stations located ahead of the driver's eye station; and then the line-of-sight was launched to check the ability of the virtual driver to see the target. The virtual driver is considered to be 


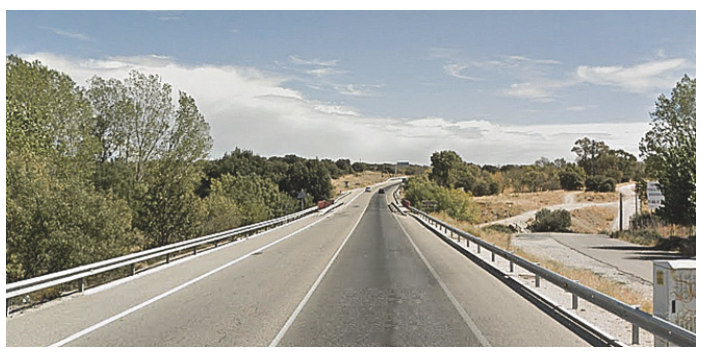

(a)

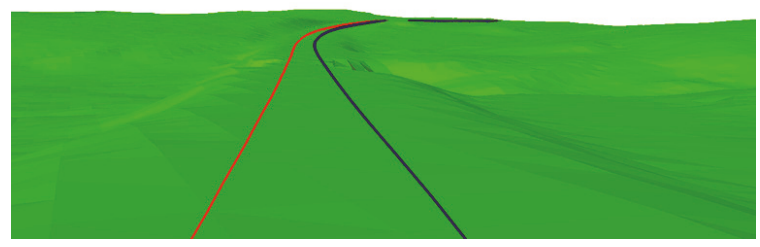

(b)

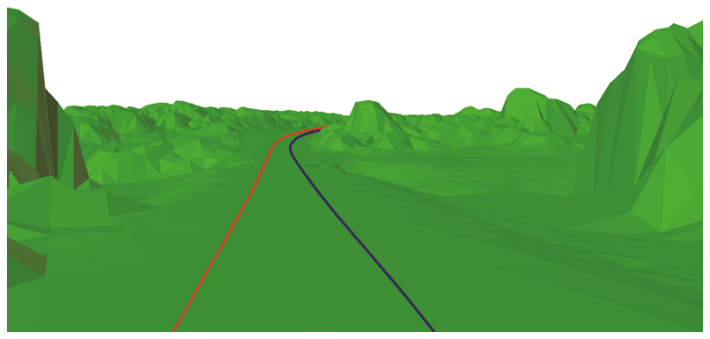

(c)

FIGURE 3: Example of 3D ASD modelling of highway M-607; (a) actual view of the highway segment, (b) 3D DTM modelling, and (c) 3D DSM modelling.

able to see the target if the line-of-sight does not intersect with the built 3D TIN surface.

Figure 3 shows an example of the 3D ASD modelling for one of the selected highways. Figure 3(a) exhibits the real view of the highways. Figure 3(b) shows the DTM surface that shapes the topography of the bare ground surface of the highway and its roadsides. Finally, Figure 3(c) illustrates the DSM surface that depicts the bare ground surface of the highway and its roadsides in addition to the relevant landscape elements above the terrain (such as trees). In Figures 3(b) and 3(c), the red and blue lines highlight the vehicle path (virtual driver position) on the roadway.

A detailed description about the used procedures for the 3D ASD modelling can be found in $[16,19]$.

4.1.3. Minimum ASD on Each Curve. For each of the three estimation methods, a minimum ASD value was assigned to each curve, which is not necessarily located within the curve. The exact position where such minimum ASD is produced is also stored to assign to the curve case the operating speed distribution of values at the beginning of the emergency stop.

4.2. Operating Speed. The use of operating speed in reliability analysis is certainly more appropriate than the consideration of design speed or posted speed. The operating speed on the roadway varies depending on the road element, its characteristics, and the driver's behavior [4]. In the deterministic approach of geometric design, the 85th percentile speed $\left(V_{85}\right)$ is utilized. However, in reliability analysis the characterization of the random variables is needed; hence the probability distribution and the 50th percentile speed $\left(V_{50}\right)$, along with statistical measures of dispersion, are considered instead of a conservative percentile value $\left(V_{85}\right)$.
In this study, the operating speed on curves whose radius is greater than $100 \mathrm{~m}$ was predicted using the model of Castro et al. [48] whereas the speed on curves with a smaller radius was derived according to Pérez Zuriaga [49]. The first model was adjusted on measurements from larger radius curves while the latter one was adjusted on smaller radius curves. Both speed prediction models provide 50th percentile speeds and were developed on highway segments in Spain.

$$
\begin{aligned}
& V_{50}=105.35-\frac{4541.98}{|R|} \text { if } R>100 \mathrm{~m}, \\
& V_{50}=\sqrt{2420.63 \cdot \log |R|-7619.89} \text { if } R<100 \mathrm{~m} .
\end{aligned}
$$

Their corresponding standard deviations are given by

$$
\begin{aligned}
& \sigma_{V}=17.03-\frac{111.25}{\sqrt{|R|}} \quad \text { if } R>100 \mathrm{~m}, \\
& \sigma_{V}=\sqrt{14.8194+5.38444 \cdot \sqrt{|R|}} \text { if } R<100 \mathrm{~m} .
\end{aligned}
$$

The speed chosen by drivers on the circular arc of the horizontal curves is assumed to follow a normal distribution whose mean is $V_{50}$ and the standard deviation is $\sigma_{V}$. The model assumes the tangent-to-curve and curve-to-curve speed transition with linear acceleration (or deceleration) variation rate [49]. On long tangents, the driver is assumed to reach the desired speed according to these rates [50]. Conversely, if the tangent is not that long, a maximum speed is reached below the desired one. The model also assumes that no forced acceleration rates are taken between circular arcs differing on their operating speeds more than the value that would enable a smooth speed transition. Instead, the driver is assumed to adjust their speed beforehand even if the previous circular arc has not been left yet. 
TABLE 2: Coefficients of friction [4].

\begin{tabular}{lccc}
\hline $\begin{array}{l}\text { Pavement } \\
\text { condition }\end{array}$ & $\begin{array}{c}\text { Mean speed } \\
(\mathrm{km} / \mathrm{h})\end{array}$ & Mean & $\begin{array}{c}\text { Standard } \\
\text { deviation }\end{array}$ \\
\hline Wet & 80.4 & 0.4192 & 0.0913 \\
Wet & 85.0 & 0.4013 & 0.0913 \\
Wet & 90.0 & 0.3826 & 0.0913 \\
Wet & 95.0 & 0.3571 & 0.0913 \\
Wet & 99.8 & 0.3498 & 0.0913 \\
Dry & All speeds & 0.8852 & 0.0949 \\
\hline
\end{tabular}

\subsection{Reliability Analysis}

4.3.1. Limit-State Function. In the context of this study, the LSF is the difference between ASD and SSD, the first one being calculated in three different ways as described in Section 4.1.

$$
\mathrm{LSF}=\mathrm{ASD}-\mathrm{SSD} .
$$

According to the current Spanish standard [6], the SSD is computed as follows:

$$
\mathrm{SSD}=\frac{V \cdot \mathrm{PRT}}{3.6}+\frac{V^{2}}{254 \cdot(f \pm i)},
$$

where $V$ is the initial speed, PRT is perception and reaction time, $f$ is the longitudinal friction, and $i$ is the grade on the vertical projection, positive if uphill and negative where downhill.

4.3.2. Random Variables. Based on (8), the reliability analysis considers three random variables: the initial speed $(V)$, the perception and reaction time (PRT), and the longitudinal friction factor $(f)$. The other two variables, the ASD and the grade ( $i$ ), are assumed to be deterministic. As mentioned earlier, the speed considered in the analysis of this paper is the operating speed which is assumed to follow a normal distribution with mean and standard deviation estimated as per (6) and (7), respectively. Along each highway segment, the operating speed is estimated at the minimum ASD station. The assumptions concerning PRT used in this study come from the results of an experiment accomplished by Lerner [51]. These assumptions are also taken as reference for PRT distribution for the National Cooperative Highway Research Program (NCHRP) [52]. Therefore, the PRT is assumed to follow a lognormal distribution with a mean of 1.5 seconds and a standard deviation of 0.4 seconds.

The longitudinal friction factor was assumed to follow a normal distribution with a mean and standard deviation that are dependent on the operating speed as shown in Table 2 [4]. For any speed value that is out of Table 2 , linear interpolation is performed to estimate the mean and the standard deviation of its corresponding friction factor. The wet surface condition was contemplated as it represents the worst case scenario for the longitudinal friction factor.

4.3.3. Probability of Noncompliance Computation. In this study, the FORM is selected for the reliability analysis.
The FORM remedies the problems associated with other reliability methods and is more efficient than sampling in terms of the number of iterations required for estimating the probability of failure [26]. The main concept of FORM is a transformation from the original space of random variables $x$ to the standard normal space of random variables $y$. In the standard normal space $y$, the LSF is linearized at the point closest to the origin. This point is called the design point, or the most probable failure point. Subsequently, the design point is the solution to the optimization problem in (0) [26].

$$
y^{*}=\operatorname{argmin} \quad\{\|y\| \mid G(y)=0\},
$$

where $y^{*}$ is the design point coordinates and $G(y)$ is the LSF in the standard normal space. By solving this optimization problem, the distance from the origin in the standard normal space to the design point $\left(y^{*}\right)$ is the reliability index $\beta$. Finally, the probability of failure (noncompliance) can be determined by using (13), where $\Phi$ is the standard normal cumulative distribution function [26].

$$
P_{\mathrm{nc}}=\Phi(-\beta) .
$$

Rt software [53] was used to compute $P_{\mathrm{nc}}$ value of the LSF shown in (10). Rt is a computer program for system reliability and optimization analysis developed by Mahsuli and Haukaas [53] at the University of British Columbia, Vancouver, Canada.

\section{Results and Discussion}

5.1. Available Sight Distance Modelling. As mentioned earlier in this paper, the ASD was calculated along the 12 selected highways in both directions. 402 curves were analyzed in both directions (804 cases) and using the three different ASD modelling methods described in Section 4.1: 2D, 3D DTM, and 3D DSM. Furthermore, the 804 selected cases were classified into 4 groups based on the existing roadside features or geometric elements that hinder driver's visibility. The four groups are as follows: (1) curves with vegetation by the roadside; (2) horizontal curves with cut side; (3) horizontal curves on a crest vertical curve; and (4) horizontal curves with none of the aforementioned features (i.e., clear highway sections). Out of the overall sample size (804 cases), the first group (vegetation) represents 463 cases, the second group (cut side) represents 147 cases, the third group (crest vertical curve) represents 129 cases, and the remaining 65 cases belong to the fourth group (clear horizontal curves). Some curves may have different obstructions depending on the direction considered. For these curves, cut side and vegetation are typically the limiting elements by the inner roadside on the direction in which the curve bends to the right, whereas a crest vertical curve is the sight restriction on the opposite direction.

By comparing the ASD results, 3D DTM ASD is equal to or greater than 3D DSM ASD in all cases. This is explained by the effect of the extra elements considered only by the DSM that reduce the ASD as described in Section 4.1.3. A $t$-test for paired samples was carried out to investigate the significance of differences between the three ASD modelling methods. 
TABLE 3: Comparison of ASD values among the three models.

\begin{tabular}{|c|c|c|c|c|}
\hline Group & ASD pair & Mean $(\mathrm{m})$ & St. dev. (m) & $p$ value \\
\hline \multirow{3}{*}{ All cases } & 2D-3D DTM & -47.4 & 100.3 & $<0.001$ \\
\hline & 2D-3D DSM & 1.5 & 48.6 & 0.372 \\
\hline & 3D DTM-3D DSM & 48.9 & 87.0 & $<0.001$ \\
\hline \multirow{3}{*}{ (1) Vegetation } & 2D-3D DTM & -69.1 & 111.6 & $<0.001$ \\
\hline & 2D-3D DSM & 10.2 & 34.5 & $<0.001$ \\
\hline & 3D DTM-3D DSM & 79.2 & 102.6 & $<0.001$ \\
\hline \multirow{3}{*}{ (2) Cut side } & 2D-3D DTM & -0.1 & 24.6 & 0.949 \\
\hline & 2D-3D DSM & 3.3 & 23.9 & 0.091 \\
\hline & 3D DTM-3D DSM & 3.5 & 3.9 & $<0.001$ \\
\hline \multirow{3}{*}{ (3) Crest } & 2D-3D DTM & 7.0 & 29.9 & 0.008 \\
\hline & 2D-3D DSM & 7.0 & 29.9 & 0.008 \\
\hline & 3D DTM-3D DSM & 0.0 & 0.0 & $1^{*}$ \\
\hline \multirow{3}{*}{ (4) Clear } & 2D-3D DTM & -108.0 & 122.9 & $<0.001$ \\
\hline & 2D-3D DSM & -75.0 & 106.7 & $<0.001$ \\
\hline & 3D DTM-3D DSM & 33.0 & 51.6 & $<0.001$ \\
\hline
\end{tabular}

${ }^{*}$ The correlation coefficient since the identical samples cannot be compared by the $t$-test.

Three pairs were considered in $t$-test: (2D-3D DTM), (2D-3D DSM), and (3D DTM-3D DSM). Table 3 outlines the results of this comparison. The $p$ values that correspond to pairs in which the difference was found to be significant at $95 \%$ confidence level are shown in bold.

For the whole sample, the results show that the difference is not significant only for the pair (2D-3D DSM). The trend of the estimated ASD shows that 3D DTM tends to overestimate the ASD comparing to the other two methods. The mean difference in the pair (2D-3D DSM) is small; however, the results are still affected by high variances.

For the first group (i.e., curves with vegetation by the roadside), a significant difference is identified for all pairs. Again, the 3D DTM overestimates the ASD comparing to the other two methods. The significant difference for the pair (2D-3D DSM) can be explained by the 3D approach. That is, the concurrence of both alignment projections (i.e., horizontal and vertical projections) and the vegetation make the $2 \mathrm{D}$ approach overestimate the ASD when compared with the 3D DSM.

For the second group (i.e., horizontal curves with cut side), only the pair that compares the two 3D methods has a significant difference. The difference is not significant for the other two pairs owing to the greater variance. Also, the mean difference in the pair (2D-3D DTM) is almost negligible, although the variance reaches 24.5 meters.

For the third group (i.e., horizontal curves on a crest vertical curve), the difference is significant for both (2D-3D) pairs. The case of (3D DTM-3D DSM) cannot be compared by the $t$-test since the ASD values are the same. This occurs because the line of sight that determines the minimum ASD lies within the roadway boundaries, and both 3D models are identical within such area. The resulting Pearson correlation coefficient equals 1 , and the mean difference equals 0 . The $2 \mathrm{D}$ method overestimates the ASD compared to the 3D methods. Presumably, this is due to the $3 \mathrm{D}$ features that are ignored by the $2 \mathrm{D}$ approach such as the effect of superelevation on the geometry, the existence of other near vertical curves, or the overlapping with a crest vertical curve. This emphasizes the importance of the 3D ASD modelling.

For the last group (i.e., clear highway sections), the results do not follow such a definite pattern as their counterparts do in the other groups. This group is the most heterogeneous as it presents the greatest differences. The clear inner-roadside allows a longer ASD in both 3D models. Moreover, the ASD values depended upon features located before or beyond the curve studied. In all pairs, a significant difference is identified. The 2D model greatly underestimates the ASD when compared to the other two 3D models. The 3D DTM overestimates the ASD in comparison with the 3D DSM model, as can be expected.

Overall, the ASD results show that there is a significant difference among the three ASD modelling methods in most cases. This emphasizes the importance of using the $3 \mathrm{D}$ modelled sight distance, particularly the 3D DSM ASD, either in highway design or during the service life as it is considered more realistic than the $2 \mathrm{D}$ one.

5.2. Effect of the ASD Modelling Approach on Safety. Considering the three ASD modelling methods and the fourgroup classification described in the previous subsection, reliability analysis was carried out to evaluate $P_{\mathrm{nc}}$ associated with each case of the selected 804 highway sections. $t$-test for paired samples was conducted to evaluate the differences between the three ASD modelling methods in terms of their corresponding $P_{\mathrm{nc}}$ using the same three pairs: (2D-3D DTM), (2D-3D DSM), and (3D DTM-3D DSM). Table 4 summarizes the results of this comparison. The $p$ values that correspond to pairs in which the difference was found to be significant at 95\% confidence level are shown in bold.

Overall, the results show that there is a significant difference in the estimated $P_{\mathrm{nc}}$ values between the three 
TABLE 4: Comparison of $P_{\mathrm{nc}}$ values among the three models.

\begin{tabular}{lcccc}
\hline Group & ASD pair & Mean & St. dev. & $p$ value \\
\hline \multirow{4}{*}{ All cases } & 2D-3D DTM & 0.105 & 0.251 & $<\mathbf{0 . 0 0 1}$ \\
& 2D-3D DSM & -0.111 & 0.277 & $<\mathbf{0 . 0 0 1}$ \\
& 3D DTM-3D DSM & -0.215 & 0.295 & $<\mathbf{0 . 0 0 1}$ \\
\hline \multirow{4}{*}{ (1) Vegetation } & 2D-3D DTM & 0.159 & 0.239 & $<\mathbf{0 . 0 0 1}$ \\
& 2D-3D DSM & -0.197 & 0.274 & $<\mathbf{0 . 0 0 1}$ \\
& 3D DTM-3D DSM & -0.355 & 0.318 & $<\mathbf{0 . 0 0 1}$ \\
\hline \multirow{4}{*}{ (2) Cut side } & 2D-3D DTM & -0.016 & 0.204 & 0.344 \\
& 2D-3D DSM & -0.049 & 0.208 & $\mathbf{0 . 0 0 5}$ \\
& 3D DTM-3D DSM & -0.033 & 0.061 & $<\mathbf{0 . 0 0 1}$ \\
(3) Crest & 2D-3D DTM & -0.042 & 0.189 & $\mathbf{0 . 0 1 2}$ \\
& 2D-3D DSM & -0.042 & 0.189 & $\mathbf{0 . 0 1 2}$ \\
& 3D DTM-3D DSM & 0.000 & 0.000 & $1^{*}$ \\
\hline \multirow{4}{*}{ (4) Clear } & 2D-3D DTM & 0.287 & 0.279 & $<\mathbf{0 . 0 0 1}$ \\
& 2D-3D DSM & 0.228 & 0.256 & $<\mathbf{0 . 0 0 1}$ \\
& 3D DTM-3D DSM & -0.059 & 0.137 & $\mathbf{0 . 0 0 1}$ \\
\hline
\end{tabular}

* The correlation coefficient since the identical samples cannot be compared by the $t$-test.

ASD modelling methods. Therefore, this emphasizes the significant implications of using different ASD modelling methods in evaluating the risk associated with a specific design. It can be noted that the models that overestimated the ASD now underestimate $P_{\text {nc }}$. The standard deviation values are as high as 0.318 , meaning that the difference in the evaluated risk is significant.

For all groups, the difference in $P_{\mathrm{nc}}$ is significant for all pairs, except only for the pair (2D-3D DTM) in the second group. Also, the pair (3D DTM-3D DSM) in the third group (crest) cannot be compared by $t$-test as it has identical $P_{\mathrm{nc}}$ values.

For the first group (i.e., curves with vegetation by the roadside), the estimated $P_{\mathrm{nc}}$ mean values indicate that the 3D DTM underestimates $P_{\text {nc }}$. The comparison (2D-3D DSM) shows that a higher risk resulted in the 3D DSM ASD model. This can be expected from the ASD results comparison.

The last group (i.e., clear highway sections) shows the greatest $P_{\mathrm{nc}}$ differences, especially in those pairs in which the 2D ASD is involved. The 2D ASD method overestimates the risk of failure by more than a $20 \%$ on average. As in most cases in the comparison of $P_{\mathrm{nc}}$ values, the corresponding shorter ASD values yield higher $P_{\mathrm{nc}}$ values.

Another noteworthy finding is that the significance of the differences in $P_{\mathrm{nc}}$ values is generally higher than the significance of the differences in the estimated ASD values. For example, the compared pairs associated with nonsignificant differences in the estimated ASD values (e.g., the 2D-3D DSM pair when comparing the whole cases) have significant differences in the estimated $P_{\mathrm{nc}}$ values at $95 \%$ confidence level. This can be explained by the nonlinear effect of the estimated ASD value on the value of $P_{\text {nc }}$ estimated through the reliability analysis. This emphasizes the importance of considering the 3D modelled sight distance, particularly the 3D DSM ASD, when evaluating the associated risk either in highway design or during the service life. Thus, the results

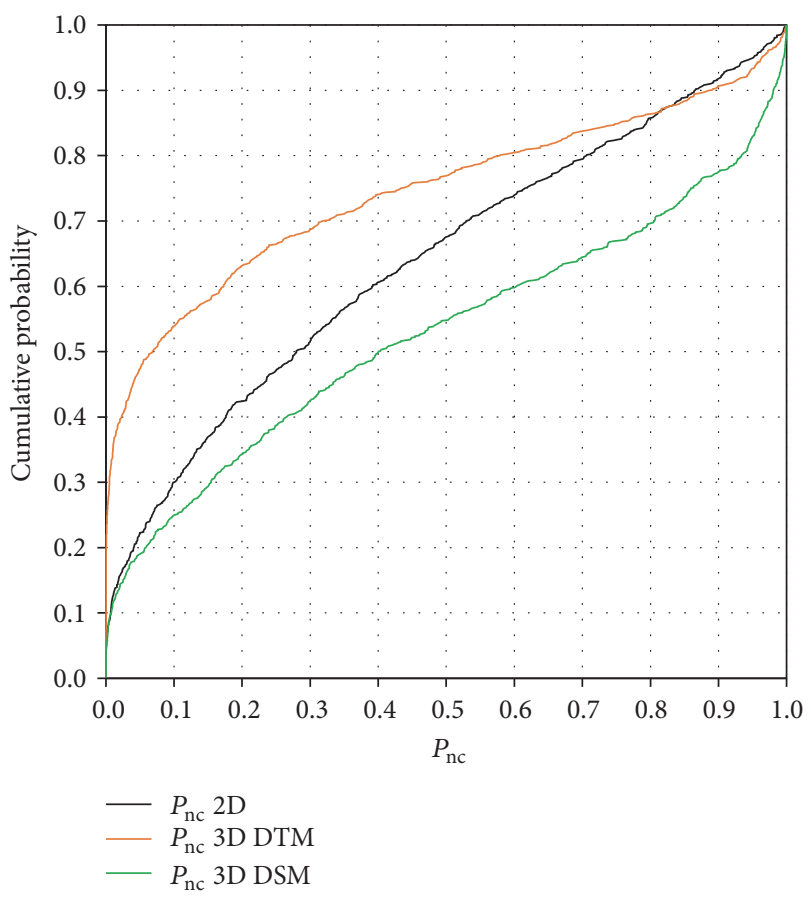

FIGURE 4: Empirical cumulative distribution of $P_{\mathrm{nc}}$.

indicate that the safety implications of the ASD modelling approach can be significant.

Figure 4 shows the empirical cumulative distribution plot of $P_{\mathrm{nc}}$ of all ASD modelling results. By comparing the cumulative distribution curves, it can be observed that small $P_{\mathrm{nc}}$ values which are close to zero are less frequent in the $3 \mathrm{D}$ ASD DSM comparing to the other two approaches. Moreover, as the cumulative frequency grows, the 3D DSM yields higher $P_{\text {nc }}$ values than the other two approaches. On the contrary, the 3D DTM approach accumulates the lowest $P_{\text {nc }}$ values. Only at the highest $P_{\mathrm{nc}}$ values (approximately over 0.84 ), the 3D DTM curve exceeds the $2 \mathrm{D}$ one. Also, the three empirical cumulative frequency curves show a frequent incidence of high $P_{\mathrm{nc}}$ values. That is, most of the analyzed cases showed to entail a high risk so that their current design does not meet the standards.

The joint frequencies for relevant $P_{\mathrm{nc}}$ values in design are exhibited in Table 5. These values correspond to the empirical cumulative frequency curves in Figure 4 . The values in Table 5 represent the share of cases whose $P_{\mathrm{nc}}$ are below a designated relevant value for each ASD model. The results give evidence that, in this range of values, the 3D DTM approach underestimates $P_{\mathrm{nc}}$ when compared to the other two approaches. The 3D DSM, on the other hand, yields the lowest number of cases complying with the designated relevant thresholds.

Figure 5 illustrates the influence of the estimated ASD value on the reliability analysis results. The three series show, approximately, the same pattern. The curves, where ASD is higher than 400 meters, have extremely small $P_{\text {nc }}$ values (close to zero). In contrast, cases with ASD below 100 meters have $P_{\mathrm{nc}}$ values range from 0 to 1. 
TABLE 5: Comparison of empirical cumulative frequencies at design $P_{\mathrm{nc}}$ values.

\begin{tabular}{lccc}
\hline$P_{\mathrm{nc}}$ & 2D & 3D DTM & 3D DSM \\
\hline 0.001 & 0.0570 & 0.2258 & 0.0498 \\
0.002 & 0.0702 & 0.2531 & 0.0573 \\
0.005 & 0.0875 & 0.2959 & 0.0911 \\
0.01 & 0.1251 & 0.3462 & 0.1137 \\
0.02 & 0.1563 & 0.3918 & 0.1393 \\
0.05 & 0.2227 & 0.4736 & 0.1900 \\
0.10 & 0.3002 & 0.5393 & 0.2496 \\
0.15 & 0.3695 & 0.5779 & 0.2938 \\
0.20 & 0.4241 & 0.6314 & 0.3429 \\
0.50 & 0.6755 & 0.7693 & 0.5482 \\
\hline
\end{tabular}

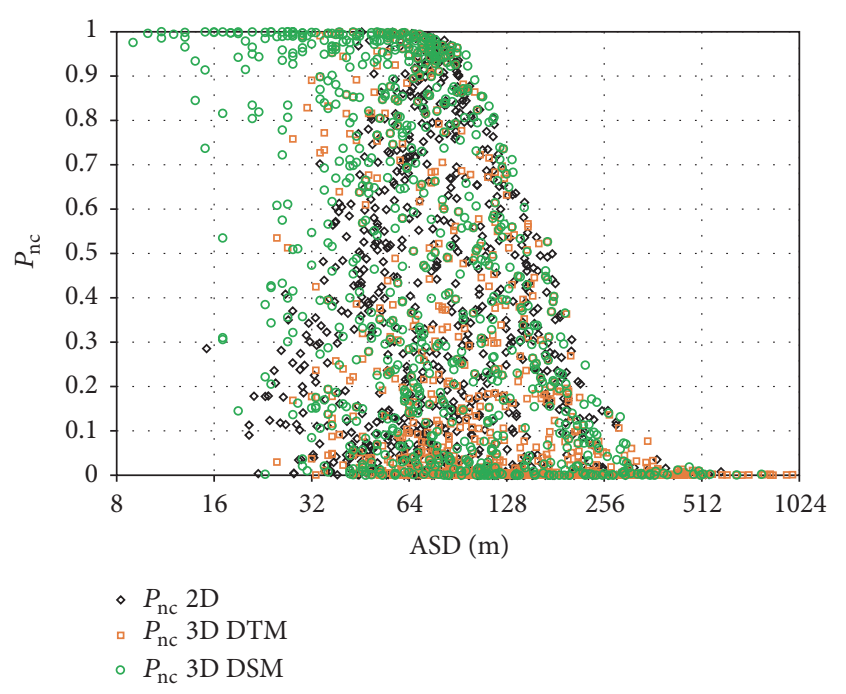

FIgURE 5: Scatter plot of ASD values and $P_{\mathrm{nc}}$.

\section{Conclusions and Future Work}

The main objective of this study is to investigate the safety implications of modelling ASD by means of reliability analysis. The ASD of 402 horizontal curves, located in twelve two-lane rural highways, is used in the analysis. Three ASD estimation methods were used: (1) 2D ASD, (2) 3D DTM ASD, and (3) 3D DSM ASD. The ASD results obtained through $2 \mathrm{D}$ and $3 \mathrm{D}$ methodologies are compared.

Overall, the ASD comparison results showed that there is a significant difference among the three ASD modelling methods in most cases. This emphasizes the importance of using the 3D modelled sight distance, particularly the $3 \mathrm{D}$ DSM ASD, either in highway design or during the service life as it is considered more realistic than the $2 \mathrm{D}$ one.

The studied curves were classified into different groups based on the existing roadside features or geometric elements that hinder driver's visibility. The resulted change in the estimated ASD between the three modelling methods under different conditions can be identified as follows:

(1) For curves with vegetation by the roadside, a significant difference is identified when comparing the three methods. The 3D DTM overestimates the ASD comparing to the other two methods. The concurrence of both alignment projections (i.e., horizontal and vertical projections) and the vegetation make the $2 \mathrm{D}$ approach overestimate the ASD when compared with the 3D DSM.

(2) For horizontal curves with cut side, only the pair that compares the two $3 \mathrm{D}$ methods has a significant difference.

(3) For horizontal curves overlapped with a crest vertical curve, the difference is significant when comparing the $2 \mathrm{D}$ approach with the $3 \mathrm{D}$ ones. The $2 \mathrm{D}$ method overestimates the ASD due to disregarding the $3 \mathrm{D}$ features such as the effect of superelevation, the existence of other near vertical curves, or the overlapping with a crest vertical curve.

(4) For curves located in clear highway sections, the results showed the greatest differences. The $2 \mathrm{D}$ model greatly underestimates the ASD when compared to the other two 3D models. The 3D DTM overestimates the ASD in comparison with the 3D DSM model.

Reliability theory was utilized to evaluate the risk level $\left(P_{\mathrm{nc}}\right)$ associated with limited sight distance for each ASD modelling method. In order to investigate the significance of the safety implications of the ASD modelling approach, $P_{\text {nc }}$ results were compared. The models that overestimated the ASD underestimated $P_{\mathrm{nc}}$. Overall, the result showed a significant difference in the estimated $P_{\mathrm{nc}}$ values between the three ASD modelling methods. This emphasizes the significant implications of using different ASD modelling methods in evaluating the risk associated with a specific design.

The significance of the differences in $P_{\mathrm{nc}}$ values is generally higher than the significance of the differences in the estimated ASD values. For example, some compared pairs are associated with a nonsignificant difference in the estimated ASD and, at the same time, have a significant difference in the estimated $P_{\mathrm{nc}}$ values at $95 \%$ confidence level. This can be explained by the nonlinear effect of the estimated ASD value on the value of $P_{\mathrm{nc}}$ estimated through the reliability analysis.

In conclusion, the results emphasize the importance of considering the 3D modelled sight distance, particularly the 3D DSM ASD, since this one is considered more realistic, when evaluating the associated risk either in highway design or during the service life. In addition, the safety implications of the ASD modelling approach can be significant.

Several future research areas can be suggested. First, an enhanced calibration of the operating speed model is desirable. It would also be advisable to assess the sensitivity analysis of the operating speed in the LSF. Second, the study assumed that the LSF input parameters are not correlated and this may not necessarily be true. Hence, a supplementary analysis on conditioned probability would enhance the applicability of results. Third, the influence of weather and pavement surface conditions requires a particular attention. Fourth, reliability results should be incorporated in safety performance functions to establish linkage to traffic safety 
and to prove that the 3D DSM ASD is the model that is closer to reality also in terms of safety. Finally, the target safety values should be defined for different highway classes or according to budgetary restrictions in construction projects.

\section{Conflicts of Interest}

The authors declare that they have no conflicts of interest.

\section{Acknowledgments}

The authors wish to acknowledge the financial support of the Spanish Ministerio de Economía y Competitividad and European Regional Development Fund (FEDER), Research Project TRA2015-63579-R (MINECO/FEDER).

\section{References}

[1] American Association of State Highway and Transportation Officials (AASHTO), A Policy on Geometric Design of Highways and Streets, AASHTO, Washington, DC, USA, 2011.

[2] X. Yan, X. Li, S. He, J. Weng, S. C. Wong, and H. Pang, "Effects of intersection field of view on emergent collision avoidance performance at unsignalized intersections: analysis based on driving simulator experiments," Journal of Advanced Transportation, vol. 50, no. 5, pp. 683-700, 2016.

[3] M. Essa, T. Sayed, and M. Hussein, "Multi-mode reliabilitybased design of horizontal curves," Accident Analysis and Prevention, vol. 93, pp. 124-134, 2016.

[4] L. Richl and T. Sayed, "Evaluating the safety risk of narrow medians using reliability analysis," Journal of Transportation Engineering, vol. 132, no. 5, pp. 366-375, 2006.

[5] M. Hussein, T. Sayed, K. Ismail, and A. Van Espen, "Calibrating road design guides using risk-based reliability analysis," Journal of Transportation Engineering, vol. 140, no. 9, Article ID 04014041, 2014.

[6] Ministerio de Fomento. Norma 3.1-IC: Trazado. Madrid, Spain: Ministerio de Fomento, 2000.

[7] Transportation Association of Canada (TAC), Geometric design guide for canadian roads, TAC, Ottawa, Ontario, Canada, 1999.

[8] T. Mauga, M. Ghanma, and K. Ahmed, "Roadside clearance limit on horizontal curves with transition arcs," Transportation Research Record, no. 2358, pp. 20-28, 2013.

[9] D. J. Lovell, "Automated calculation of sight distance from horizontal geometry," Journal of Transportation Engineering, vol. 125, no. 4, pp. 297-304, 1999.

[10] K. Ismail and T. Sayed, "New algorithm for calculating 3D available sight distance," Journal of Transportation Engineering, vol. 133, no. 10, pp. 572-581, 2007.

[11] Y. Hassan, S. M. Easa, and A. O. Abd El Halim, "Design considerations for combined highway alignments," Journal of Transportation Engineering, vol. 123, no. 1, pp. 60-68, 1997.

[12] R. Lamm, B. Psarianos, and T. Mailaender, Highway design and traffic safety engineering handbook, McGraw-Hill, Columbus, Ohio, Canada, 1999.

[13] Y. Hassan, S. M. Easa, and A. O. Abd El Halim, "Analytical model for sight distance analysis on three-dimensional highway alignments," Transportation Research Record, vol. 1523, no. 1, pp. 1-10, 1996.

[14] Forschungsgesellschaft für Straßen- und Verkehrswesen (FGSV), Hinweise zur Visualisierung von Entwürfen für außerörtliche Straßen (H ViSt), FGSV-Verlag, Bonn, Germany, 2008.

[15] M. Castro, L. Iglesias, J. A. Sánchez, and L. Ambrosio, "Sight distance analysis of highways using GIS tools," Transportation Research Part C: Emerging Technologies, vol. 19, no. 6, pp. 9971005, 2011.

[16] M. Castro, J. A. Anta, L. Iglesias, and J. A. Sánchez, "GISbased system for sight distance analysis of highways," Journal of Computing in Civil Engineering, vol. 28, no. 3, Article ID 04014005, 2014.

[17] J. M. Campoy-Ungría, A. García, A. M. Perez-Zuriaga, and F. J. Camacho-Torregrosa, "Nueva metodología para la obtención de visibilidades disponibles en carreteras a partir de datos LiDAR mobile," Rutas Técnica, vol. 153, pp. 16-23, 2012.

[18] J. Tarel, P. Charbonnier, F. Goulette, and J. Deschaud, "3D Road Environment Modeling Applied to Visibility Mapping: An Experimental Comparison," in Proceedings of the 16th International Symposium on Distributed Simulation and Real Time Applications (DS-RT), pp. 19-26, Dublin, Ireland, October 2012.

[19] M. Castro, S. Lopez-Cuervo, M. Paréns-González, and C. de Santos-Berbel, "LIDAR-based roadway and roadside modelling for sight distance studies," Survey Review, vol. 48, no. 350, pp. 309-315, 2016.

[20] P. L. Olson, D. E. Cleveland, P. S. Fancher, L. P. Kostyniuk, and L. W. Schneider, "Parameters affecting stopping sight distance," National Cooperative Highway Research Program Report, 1984.

[21] K. L. Fink and R. A. Krammes, "Tangent length and sight distance effects on accident rates at horizontal curves on rural two-lane highways," Transportation Research Record, vol. 1500, no. 1, pp. 162-168, 1995.

[22] B. Steinauer, R. Trapp, and E. Böker, "Verkehrssicherheit in Kurven auf Autobahnen," Straßenverkehrstechnik, vol. 46, no. 8, pp. 389-393, 2002.

[23] K. Fitzpatrick, D. B. Fambro, and A. M. Stoddard, "Safety effects of limited stopping sight distance on crest vertical curves," Transportation Research Record, no. 1701, pp. 17-24, 2000.

[24] S. E.-B. Ibrahim and T. Sayed, "Developing safety performance functions incorporating reliability-based risk measures," Accident Analysis and Prevention, vol. 43, no. 6, pp. 2153-2159, 2011.

[25] F. P. D. Navin, "Safety factors for road design: Can they be estimated?" Transportation Research Record, vol. 1280, no. 1, pp. 181-189, 1990.

[26] T. Haukaas, "Mean-value first-order second moment method (MVFOSM)," InRisk 2012, http://inrisk.sites.olt.ubc.ca/files/ 2012/11/MVFOSM.pdf.

[27] A. Chen, P. Kasikitwiwat, and C. Yang, "Alternate capacity reliability measures for transportation networks," Journal of Advanced Transportation, vol. 47, no. 1, pp. 79-104, 2013.

[28] M. Ben-Akiva, M. Hirsh, and J. Prashker, "Probabilistic and economic factors in highway geometric design," Transportation Science, vol. 19, no. 1, pp. 38-57, 1985.

[29] A. Faghri and M. J. Demetsky, "Reliability and risk assessment in the prediction of hazards at rail-highway grade crossings," Transportation Research Record, no. 1160, pp. 45-51, 1988.

[30] S. M. Easa, "Reliability-based design of sight distance at railroad grade crossings," Transportation Research Part A, vol. 28, no. 1, pp. 1-15, 1994.

[31] S. M. Easa, "Reliability approach to intersection sight distance design," Transportation Research Record, vol. 1701, no. 1, pp. 42$52,2000$. 
[32] F. P. Navin, "Reliability indices for road geometric design," Canadian Journal of Civil Engineering, vol. 19, no. 5, pp. 760766, 1992.

[33] T. Echaveguren, M. Bustos, and H. de Solminihac, "Assessment of horizontal curves of an existing road using reliability concepts," Canadian Journal of Civil Engineering, vol. 32, no. 6, pp. 1030-1038, 2005.

[34] J. E. Khoury and A. G. Hobeika, "Assessing the risk in the design of passing sight distances," Journal of Transportation Engineering, vol. 133, no. 6, pp. 370-377, 2007.

[35] M. Sarhan and Y. Hassan, "Three-dimensional, probabilistic highway design sight distance application," Transportation Research Record, no. 2060, pp. 10-18, 2008.

[36] C. De Santos-Berbel and M. Castro, "Stopping-sight-distance simulation using a new probabilistic approach," in Proceedings of the 5th International Symposium on Highway Geometric Design, Vancouver, British Columbia, Canada, 2015.

[37] K. Ismail and T. Sayed, "Risk-based highway design: Case studies from British Columbia, Canada," Transportation Research Record, no. 2195, pp. 3-13, 2010.

[38] K. Ismail and T. Sayed, "Risk-optimal highway design: Methodology and case studies," Safety Science, vol. 50, no. 7, pp. 15131521, 2012.

[39] S. E. Ibrahim, T. Sayed, and K. Ismail, "Methodology for safety optimization of highway cross-sections for horizontal curves with restricted sight distance," Accident Analysis and Prevention, vol. 49, pp. 476-485, 2012.

[40] C. Llorca, A. Moreno, T. Sayed, and A. García, "Sight distance standards based on observational data risk evaluation of passing," Transportation Research Record, no. 2404, pp. 18-26, 2014.

[41] S. C. Himes and E. T. Donnell, "Reliability approach to horizontal curve design," Transportation Research Record, vol. 2436, pp. 51-59, 2014.

[42] A. Musunuru and R. J. Porter, "Reliability-based geometric design approach for selecting basic number of freeway lanes," Transportation Research Record, vol. 2436, pp. 70-80, 2014.

[43] K. You, L. Sun, and W. Gu, "Reliability-based risk analysis of roadway horizontal curves," Journal of Transportation Engineering, vol. 138, no. 8, pp. 1071-1081, 2012.

[44] K. You and L. Sun, "Reliability analysis of vehicle stability on combined horizontal and vertical alignments: Driving safety perspective," Journal of Transportation Engineering, vol. 139, no. 8, pp. 804-813, 2013.

[45] J. Shin and I. Lee, "Reliability-based vehicle safety assessment and design optimization of roadway radius and speed limit in windy environments," Journal of Mechanical Design, Transactions of the ASME, vol. 136, no. 8, Article ID 081006, 2014.

[46] A. Osama, T. Sayed, and S. Easa, "Framework for evaluating risk of limited sight distance for permitted left-turn movements: Case study," Canadian Journal of Civil Engineering, vol. 43, no. 4, pp. 369-377, 2016.

[47] C. Kraemer, J. M. Pardillo, S. Rocci, M. G. Romana, V. Sánchez Blanco, and M. A. Del Val, Ingeniería de carreteras, McGrawHill, Madrid, Spain, 2009.

[48] M. Castro, L. Iglesias, R. Rodríguez-Solano, and J. A. Sánchez, "Highway safety analysis using geographic information systems," Proceedings of the Institution of Civil Engineers: Transport, vol. 161, no. 2, pp. 91-97, 2008.

[49] A. M. Pérez Zuriaga, Caracterización y modelización de la velocidad de operación en carreteras convencionales a partir de la observación naturalística de la evolución de vehículos ligeros. [Doctoral, thesis], Universidad Politécnica de Valencia, Valencia, Spain, 2012.

[50] J. L. Cardoso, "Relations between accident frequency and speed consistency in Portuguese two-lane/two-way highways links," in Proceedings of the International Symposium on Highway Geometric Design Practice, pp. 1-10, Boston, Massachusetts, USA, 1995.

[51] N. Lerner, "Age and driver perception-reaction time for sight distance design requirements," in Proceedings of the Institute of Transportation Engineers 65th Annual Meeting, pp. 624-628, Colorado, Denver, Colorado, 1995.

[52] M. A. Brewer, Recent Roadway Geometric Design Research for Improved Safety and Operations, National Academies Press, Washington, D.C., USA, 2012.

[53] M. Mahsuli and T. Haukaas, "Computer program for multimodel reliability and optimization analysis," Journal of Computing in Civil Engineering, vol. 27, no. 1, pp. 87-98, 2013. 


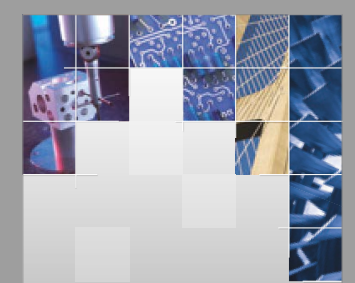

\section{Enfincering}
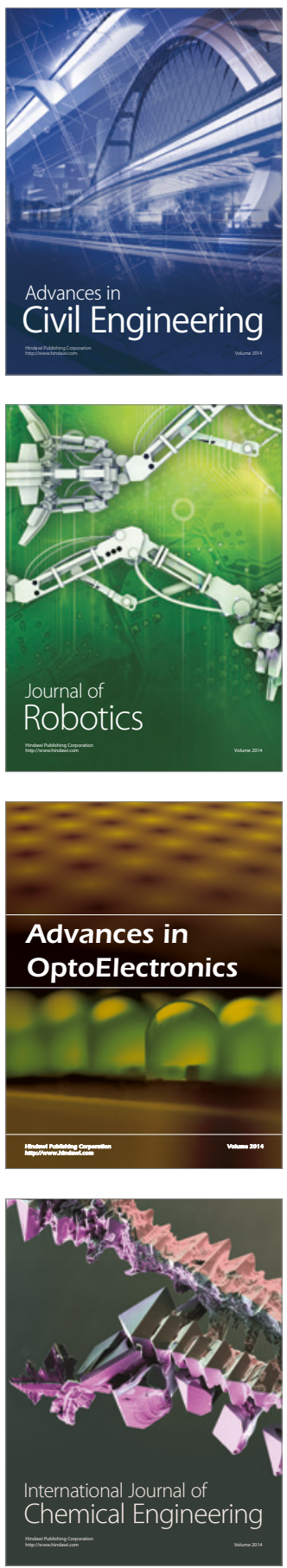

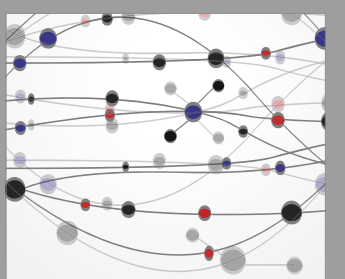

The Scientific World Journal

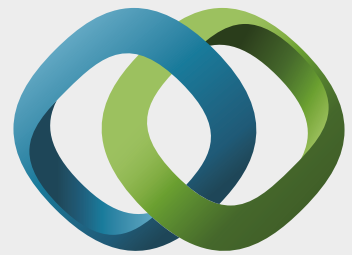

\section{Hindawi}

Submit your manuscripts at

https://www.hindawi.com
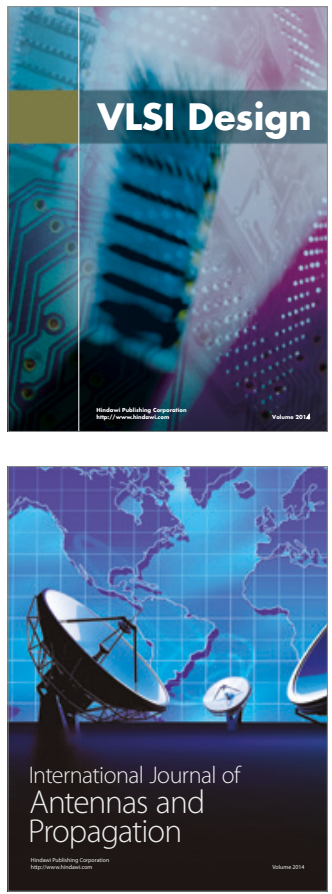

\section{Rotating}

Machinery
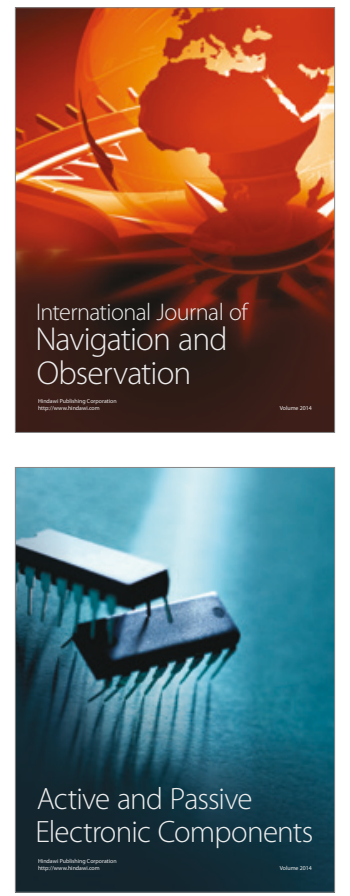
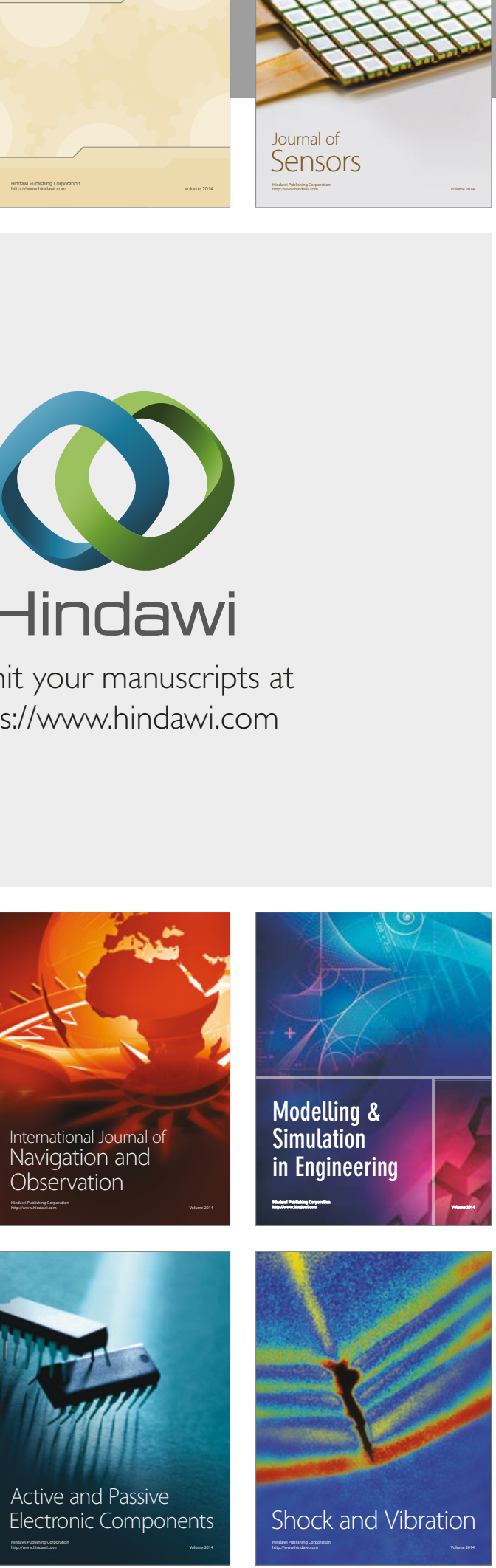
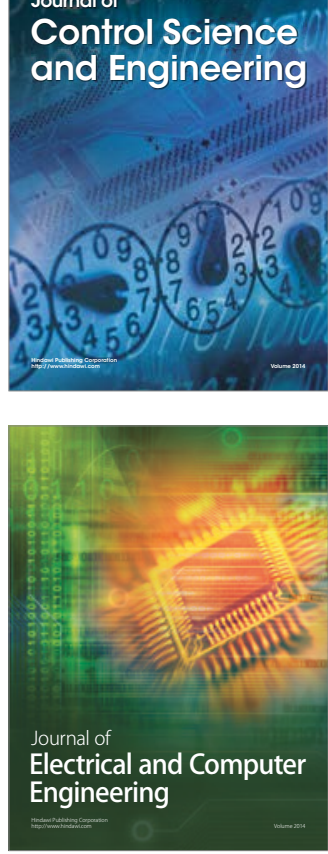

Distributed

Journal of

Control Science

and Engineering
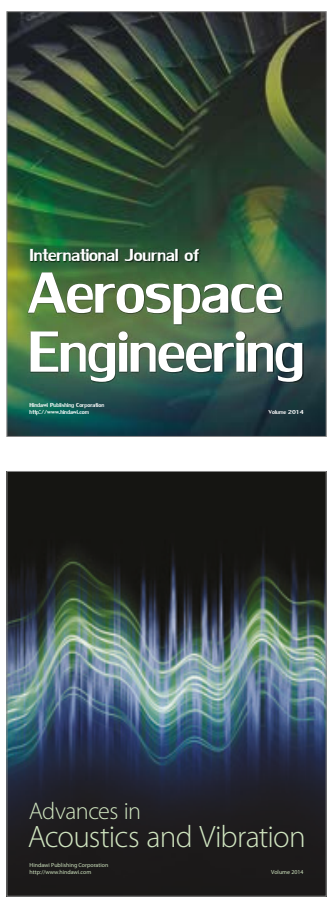

Sensor Networks 